\title{
Carta al editor
}

\section{Percepción visual en pacientes esquizofrénicos}

\author{
E. Bodner ${ }^{1}$ y Y. Barak ${ }^{2}$ \\ 'Departamento de Psicología, Universidad Bar-Ilan, Ramat-Gan, Israel; ${ }^{2}$ Centro de Salud Mental Abarbnel, \\ St. Bat-Yam, Israel
}

Desde la década de 1960 , se ha reconocido e investigado la sensibilidad de los pacientes esquizofrénicos a los estímulos no verbales. Estos pacientes muestran dificultades para diferenciar entre lo que «ven» de hecho y lo que «deberían» ver. Es posible explicar la sensibilidad e inquietud que experimentan los esquizofrénicos con respecto a ciertos estímulos por su relación con experiencias vitales y condicionamientos sociales o con déficit patofisiológicos más básicos. En efecto, R. Cooper demostró en 1960 diferencias significativas entre esquizofrénicos y normales en medidas objetivas de percepción.

Uno de los sólidos parámetros que diferencian esquizofrénicos paranoides frente a no paranoides es en qué medida exploran el entorno buscando estímulos (Silverman, 1964). Sus estudios utilizaron un diseño en el que paranoides y no paranoides fueron examinados en una tarea de exploracióncontrol. Brevemente, los sujetos tenían que ajustar una zona circular de luz a cada uno de tres discos de distinto tamaño sostenidos manualmente. Los esquizofrénicos paranoides hicieron un gran uso significativamente de la exploración, mientras que los no paranoides dieron pruebas de una exploración mínima. Estudios adicionales (McCormick y Broekem, 1978) demostraron que los esquizofrénicos paranoides subestiman el tamaño de los estímulos visuales. Pensamos que estas líneas de investigación convergen en una manera por la cual una exploración extensa produce una falsa disminución en el tamaño percibido de los estímulos visuales presentados a los esquizofrénicos paranoides.

Recientemente, Aharonovich et al (1993) sometieron a prueba la hipótesis de que la disfunción hemisférica varía entre esquizofrénicos paranoides y no paranoides. Se interpretó que sus resultados indicaban asociaciones específicas a la modalidad (visual) de la esquizofrenia paranoide con disfunción del hemisferio izquierdo. Sin embargo, los estímulos utilizados para examinar a los sujetos no tenían en cuenta el cuerpo de datos que apunta hacia el papel de la exploración y la estimación del tamaño en estos pacientes. Está por ver si los diversos estudios centrados en la percepción visual en esquizofrénicos producirán una teoría coherente. Hasta ese momento, el diseño de experimentos no debe dejar de tener en cuenta los parámetros de exploración y estimación del tamaño para una interpretación más clara de los resultados.

Aharanovich E, Karny N, Nachson I. Visual field processing in paranoid and non-paranoid schizophrenics. Eur Psychiatry 1993; 8: 301-7.

McCornick D, Broekem V. Size estimation, perceptual recognition and cardiac rate response in acute paranoid and non-paranoid schizophrenics. J Abn Psychol 1978; 87: 385-98.

Silverman J. Scanning-control mechanism and cognitive filtering in paranoid and non-paranoid schizophrenia. J Consult Psychol 1964; 28 (5): 385-93.

Bodner E, Barak Y. Visual percepcion in schizophrenic patients. Eur Psychiatry 1995; 10: 320 


\title{
Libro blanco sobre «Calidad Asistencial de la Depresión en España»
}

\author{
El pasado 1 de febrero se celebró en Madrid una Mesa Redonda para la presentación del Estudio Sociosanitario: \\ Libro Blanco sobre "La Calidad Asistencial de la Depresión en España". Para su realización se cuenta con la \\ colaboración de las Sociedades Españolas de Psiquiatría y de Medicina General, bajo el patrocinio de \\ Laboratorios Juste S.A.Q.F.
}

La reunión estuvo presidida por el Prof. D. Juan José López-lbor, con la participación de destacados psiquiatras y representantes de la Asistencia Primaria, que determinaron la finalidad, el contenido y la metodología del proyecto.

El primer objetivo marcado fue conseguir la integración de psiquiatras y médicos de Atención Primaria en el Estudio, en base al intercambio de opiniones, información, etc., a través de la creación del Primer Panel Juste de Calidad Asistencial de la Depresión en España, al que se invitará a colaborar a dichos profesionales mediante la aportación de sus conocimientos y experiencia.

El Estudio pretende analizar desde distintos puntos de vista (psiquiatras, médicos de Atención Primaria y pacientes) el estado actual de la situación asistencial al paciente deprimido. Para ello, estudiará la posibilidad de aplicar al mismo las Normas Internacionales de Calidad (ISO 9000), lo que hace que el trabajo se considere como pionero, ya que es la primera vez que se aplican en España, dentro del campo de la Sanidad, los citados modelos de referencia.

Otro de los objetivos planteados será delimitar los niveles de colaboración entre Psiquiatría y Atención Primaria, ya que existe la convicción de que algunos cuadros mentales

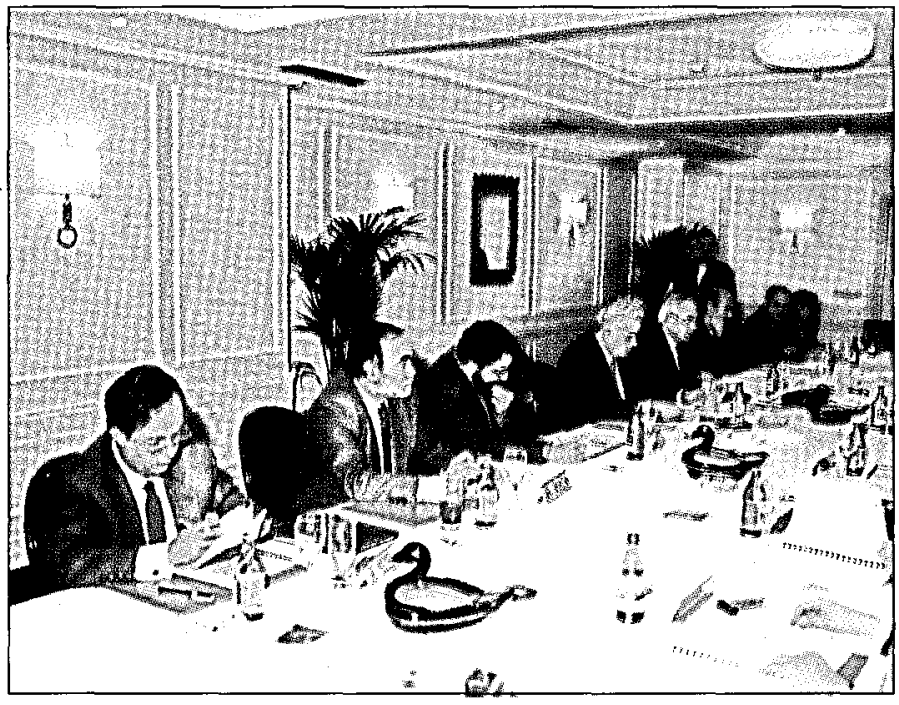

son susceptibles de mejora si son atendidos en el nivel sanitario más adecuado.

En suma, el objetivo general del Estudio consistirá en disponer, probablemente por primera vez en España, de una evaluación de los procesos de diagnóstico, tratamiento y seguimiento de los pacientes depresivos en Atención Primaria y Especializada, lo que incrementará las posibilidades de mejora de la calidad asistencial existente.

Además se recogerá información acerca de:

- Demanda de asistencia en este trastorno psiquiátrico. Población asistida y población no asistida.

- Percepción y valoración de la gravedad que tiene la depresión en la población general.

- Circuitos que sigue el paciente. Areas de cobertura: relaciones y dependencias organizativas en Atención Primaria y Especializada.

- Seguimiento de estos pacientes: medios diagnósticos y terapias empleadas; niveles de eficacia del tratamiento farmacológico y no farmacológico; cumplimiento del tratamiento por parte del paciente.

El Estudio será realizado por el Gabinete de Estudios Sociológicos Bernard Krief, que procederá al análisis descriptivo de toda la información recogida y a partir de este informe se editará el Libro Blanco sobre «Calidad Asistencial de la Depresión en España» y la colaboración entre el psiquiatra y la Asistencia Primaria para su mejora.

En la actualidad existe un consenso sobre el serio problema que representa la depresión, no sólo a nivel del especialista, sino también para el médico de Atención Primaria. La elevada frecuencia de esta patología, junto con la interferencia de la misma sobre el nivel de vida físico, social y familiar, hacen que la depresión tenga una entidad propia y susceptible de ser abordada en un Estudio de estas características. Para los Laboratorios Juste S.A.Q.F. el patrocinio de este proyecto constituye un paso más en la colaboración científica, técnica y humana, que desde hace 75 años viene manteniendo con el mundo sanitario español. 


\section{Agenda}

\section{Psiquiatría Europea: Una fuerza para el futuro, Congreso Conjunto, Londres, 7-12 de julio de 1996}

El 8. ${ }^{\circ}$ Congreso de la Asociación de Psiquiatras Europeos (AEP) se celebrará en Londres, del 7 al 12 de julio de 1996, y se combinará con la Reunión Anual del Real Colegio de Psiquiatras. La AEP se ha convertido cada vez más en el foro principal para intercambio científico entre psiquiatras de los diferentes países europeos y el último congreso, celebrado en Copenhague en 1994, atrajo a más de 2.500 psiquiatras. Se anticipa que el $8^{\circ}$ congreso en Londres tendrá un éxito aún mayor.

El congreso tendrá simposios organizados, comunicación libre y simposios de investigación reciente, debates en mesa redonda y presentaciones de pósters. Además, habrá un amplio programa social. También, psiquiatras de diferentes países tendrán la oportunidad de reunirse para discutir las áreas de interés mutuo referentes al desarrollo de cuestiones profesionales en diferentes países y las cuestiones semipolíticas con respecto a la psiquiatría en diversos países.

Se puede obtener información adicional de: AEP Congress Secretariat, Royal College of Psychiatrist, 17 Belgrave Square, London SW1X 8PG, Reino Unido. El plazo de presentación de resúmenes para el congreso es enero de 1996. Los que deseen hacer otras propuestas para el programa científico deben ponerse en contacto con el Prof. R. Murray, Presidente del Comité Científico, en la Secretaría del Congreso.

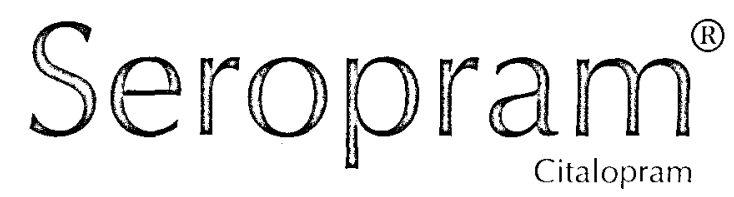

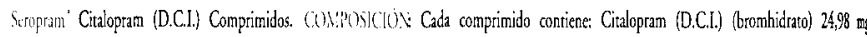

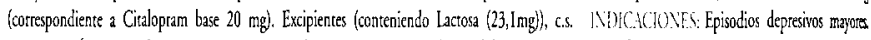

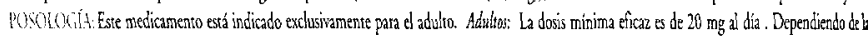

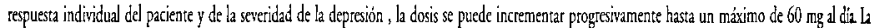

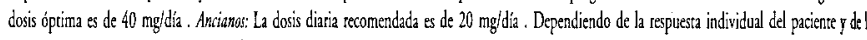

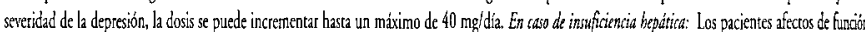

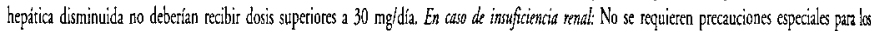

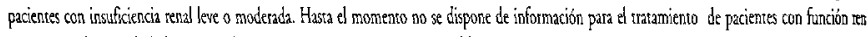

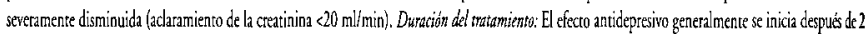
4 semanas de iniciado el tratamiento. El tratamiento con anidepresivos es sintomático y. pot tanto, debe continuarse durante un periodo de tiempo apropiado, generalmence durante seis meses, a fin de prevenir tecádas. NNo existen estudios sobre la prevención. de recurrencias. FORVIA DE

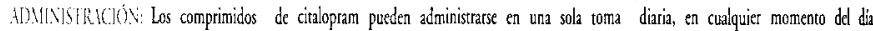

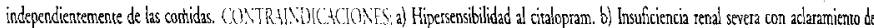
creacinina inferior a $20 \mathrm{~m} /$ /minuro, ance la alsencia de datos. c) Niños de menos de 15 años de edad: no se dispone de datos. d) Asociación con los IMHO no selectivo e IMAO selectivos B (véese inereacciones con otros medicamentoss. e) Contraindiaciones relarivas: asciaciones con los IMAO selectivos A.

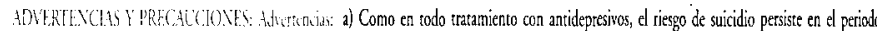
inicial del tratamientọ en los pacientes depresivos, porque la supresión de la jabibibión psicomotora puede preceder a la acción antidepresiva propiamente dicha.. b) Dado que a inicio de tratamiento aparecen insonnio o nerviosismo, se puede considerat una disminución de la dosis o un tratamiento sedane asociado hasta la mąjoria del episodio deptesivo. c) Esta especialidad contiene lactosa. Se han descrito casos de intolerancia a este componente en nifóos y

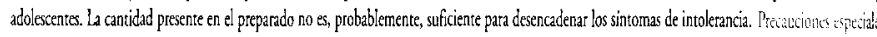

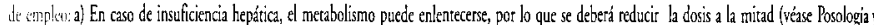
forma de administración), b) En caso de crisis maniaca debe inerrumpirse el tratamiento con ciralopram y prescribisse un neurolépyoso sedante. c) La

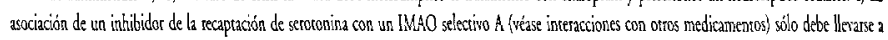

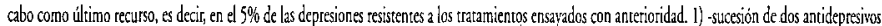
de mecanismo diference y prescritos en monoternpia. 2) -asociación de un antidepresivo con licio. Si estas tentativas fracesan essa asocicióón puede considerarse como último recurso. pero impone un seguimicnto estremadamente riguroso del pacience, teniendo en cuenta el riesgo de sindrone serotoninéegico" (véase más adelante) a que esta expuesso. d) En los pacientes que tengan ancecederntes de epilepsia es prudente reforzar la vigilancia

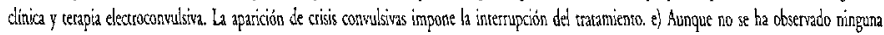

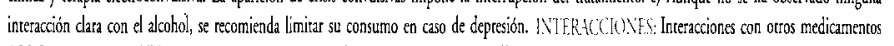

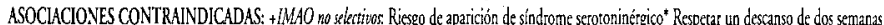
encre la retirada de un MPAO y el inicio del rracamiento con ciraopram, y de al menos ína semana entre la reirada de ciralopram y el inicio de un

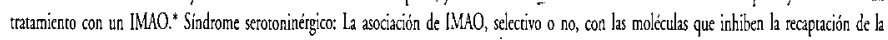

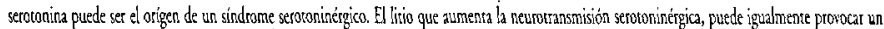
sindrome serotoninérgico con los intibidores de la recaptación de la secooronina, pero de mancera mis atenuada. Dicho sindrome se manifiesta por la aparición (en agún caso brusca), simultảnea o sucesiva de un conjunto de sintomas que pueden hacer necesaria la hospitalización o incluso producir la

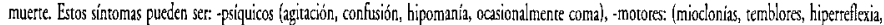

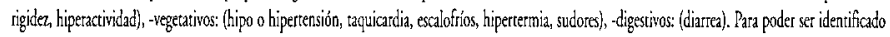

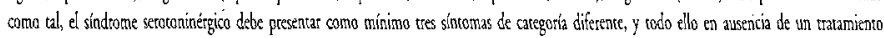
neuroléptico recienternente asociado o de un aumenco recience de la dosis de un treatamiento neurnlépico ascciado, teniendo en cuenta las similirudes dínicas con el sindrome maligno de los neurolépricos. El essricto respeco de la posslogia indicada conssinuye un factor esencial en la prevención de la

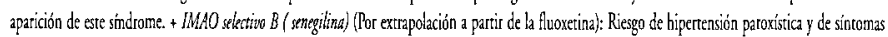

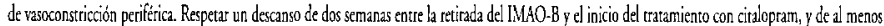
una semana entre la ectirada del citalopram y el inicio de un tratemicnto por IMAO-B. ASOCHACOON NO RECOMENDABLE: + HMAO selectivaA

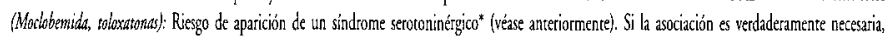
realizar una vigilancia dlínica muy estrectha. (véase Precauciones de cmpleo). ASOCLACIONES QUE PRECISAV PRECALCIONES DE EMPLEO: + Carbamazepina (por entrapolacioin a partir de la fluxxtina y de if favoxamina). Aurnento de los niveles de carbamazepina con signos de sobredosis. Vigilancia clinica con control de las concentraciones plasmíticas de carbanazepina y posible reducción de la posología de cabbamazepina duranne elt

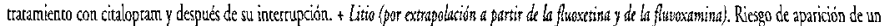

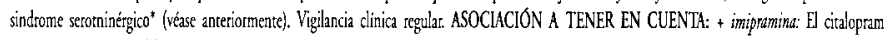

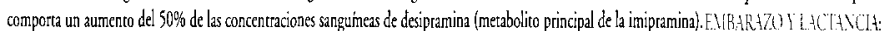
La escasez de observaciones clinicas disponibles impone la prudencia en la mujer embarazada y durance la lactancia (véase Daros preclinicos de seguridad).

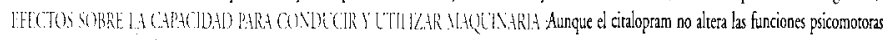

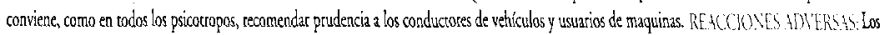

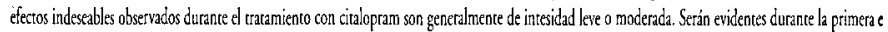
incliso las dos primeras semanas del tratamienco, $y$ posteriormente desaparecen al mejorar el episodio depresivo. Se han enconcrado en monoterapia o en

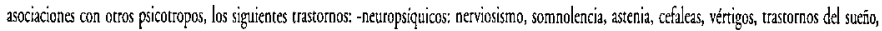

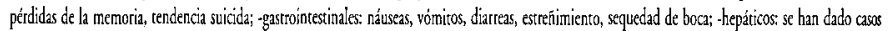

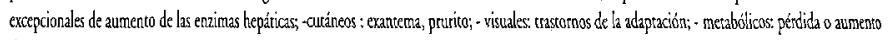
de pesoi - cardiovacsulares: taquicardia hiporensión orcoscácica, bradicardia en los pacientes que tienen una frecuencia cardiaca baja; -de la libido; - de ha

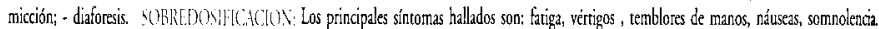
Durante las tentarivas de intoxicación voluntaria con una mezala de medicamentos, asociada o no con el a acohol, el citalopram. no parece provocar $h$ aparición de una sintomarológia clínica especifica, salvo con los IMAO sclectivos A (vésese Interacciones con otros medicamentos y precauciones de

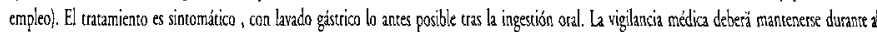

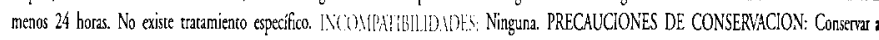

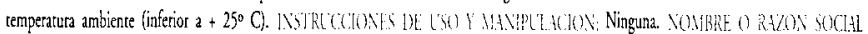

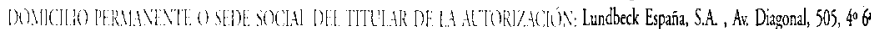

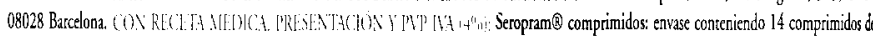

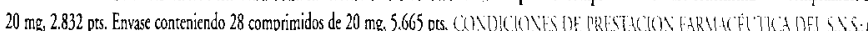

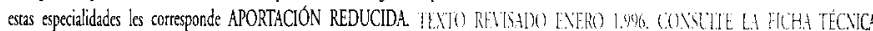

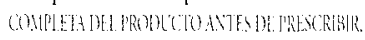

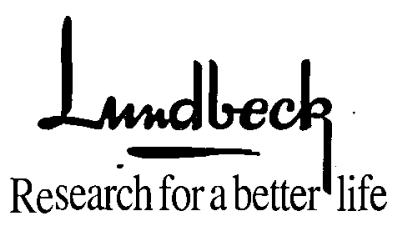




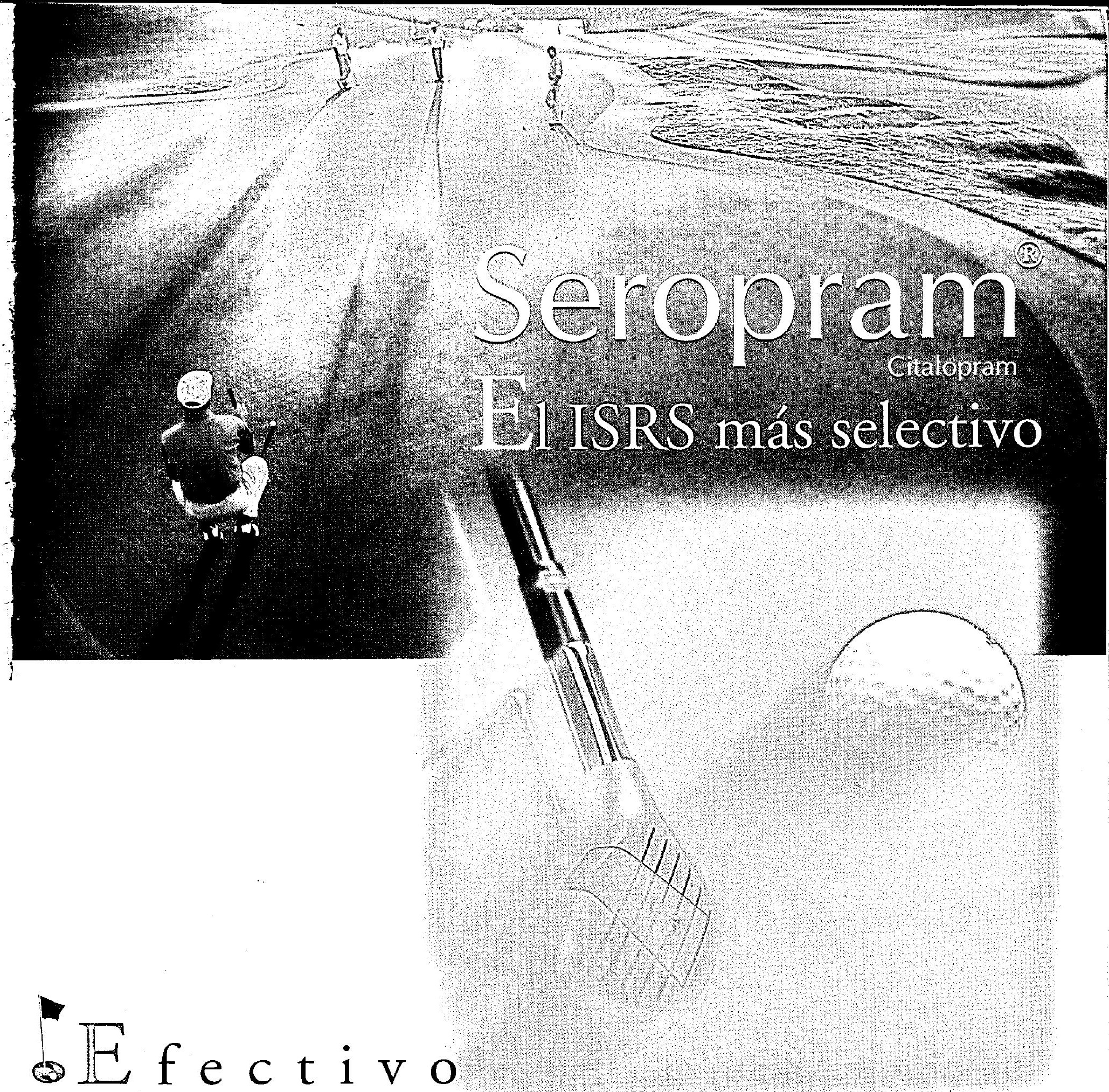




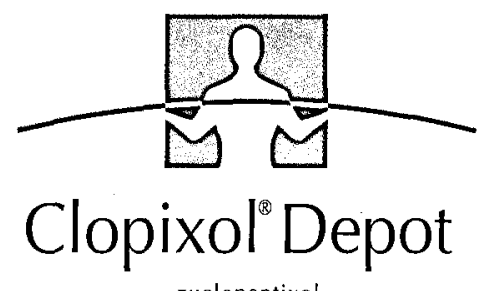

zuclopentixol

CLOPIXOI ${ }^{(}$Depot. Zuclopentixol (DCI). Clopixol $\left.{ }^{(}\right)$Depot, inyectable. COMPOSICION: Cada ampolla de $1 \mathrm{ml}$ contiene: zuclopentixol (DCl) decanoato, 200 mg. Excipiente: aceite vegetal. INDICACIONES: Tratamiento de la esquizofrenia crónica y subcrónica especialmente en pacientes en los que existe dificultad de establecer un cumplimiento por vía oral. POSOLOGIA: Adultos: La dosis y el intervalo de administración deberá ajustarse individualmente, para alcanzar una supresión máxima de los síntomas psicóticos con un mínimo de efectos indesea dos. En el tratamiento de mantenimiento las dosis oscilarán entre 200 y $400 \mathrm{mg}$ cada 2 a 4 semanas. Algunos pacientes pueden necesitar dosis superiores 0 intervalos inferiores entre las dosis. La' dosis máxima recomendada es de $000 \mathrm{mg}$ (3ml) por semana. Cuando se cambie el tratamiento de zuclopentixol oral o ace tato de zuclopentixol intramuscular a tratamiento de mantenimiento con decanoato de zuclopentixol se seguirán las siguientes directrices: 1) Cambio de zuclopentixol oral a decanoato de zuclopentixol: $25 \mathrm{mg} /$ día por vía oral equivaen a $200 \mathrm{mg}$ de decanoato de zuclopentixol cada 2 semanas; $25 \mathrm{mg} / \mathrm{dia}$ por vía oral equivalen a $400 \mathrm{mg}$ de decanoato de zuclopentixol cada 4 semanas. 2) Cambio de acetato de zuclopentixol a decanoato de zuclopentixol: simultáneamente con la (última) inyección de acetato de zuclopentixol $(100 \mathrm{mgl}$, se administrarán $200-400 \mathrm{mg}(1-2 \mathrm{ml})$ de decanoato de zuclopentixol $200 \mathrm{mg} / \mathrm{ml}$. Las inyecciones de decanoato de zuclopentixol se repetirán cado dos semanas. Algunos pacientes pueden necesitar dosis superiores o intervalos inferiores entre las dosis. El acetato de zuclopentixol y el decanoato de zuclopentixol pueden mezclarse en una jeringa y administrarse como una sola inyección intramuscular [coinyección). Los pacientes que hayan seguido tratamiento con otros neurolépticos depot recibirán una dosis de decanoato de zuclopentixol según la relación: $200 \mathrm{mg}$ de decanoato de zuclopentixol equivalen a $25 \mathrm{mg}$ de decanoato de flufenacina. Las subsiguientes dosis de decanoato de zuclopentixol y los intervalos entre las inyecciones se ajustarán según la respuesta del paciente. Pautas posológicas especiales: Reducción de la función renal: El decanoato de zuclo pentixol puede administrarse a las dosis habituales en pacientes con reducción de la función renal. Reducción de la función hepática: Se recomienda una dosificación cuidadosa $y$, si es posible, determinaciones de los niveles sericos del fármaco. Niños: No se recomienda su utilización en niños, debido a la falta de ex periencia clínica. FORMA DE ADMINISTRACION: El decanoato de zuclopentixol se administra por inyección intramuscular en el cuadrante superoexterno de la re gión glútea. Cuando el volumen de inyección sea superior a $2 \mathrm{ml}$. deberó repartirse en dos zonas de inyección diferentes. Por tratarse de un preparado inyectoble con vehículo oleoso, se recomienda efectuar una aspiración antes de administrar el medicamento, para así descartar la posibilidad de inyección intravascular. La tolerabilidad local es buena. CONTRAINDICACIONES: Hipersensibilidad al principio activo o a los tioxantenos en general. Insuficiencia circulatoria, cualquier depresión del sistema nevvioso central independientemente de su origen lintoxicaciones por alcohol, barbitúricos u opiáceos), en estados comatosos, discrasias sanguíneas, feocromocitoma. ADVERTENCIAS Y PRECAUCIONES: Al igual que otros neurolépticos el zuclopentixol debe utilizarse con precaución en pacientes con enfermedades orgánicas cerebrales, enfermedad de Parkinson, desór denes convulsivos(epilepsia), enfermedad cardiovascular o arritmia, enfermedad hepática o respiratoria avanzada. Si bien no hay ninguna evidencia que sugiera que el empleo de zuclopentixol puede dar origen a problemas especiales en pacientes con hipotiroidismo, tirotoxicosis, miastenia gravis o hipertrofia prostati $\mathrm{ca}$ en tales casos deben observarse las mismas precauciones que con el resto de neurolépticos. De igual manera deberá utilizarse con precaución en pacientes ancianos en situación crítica $O$ con riesgo de padecer hipotermia. $Y$ en aquellos pacientes con historia personal o familiar de glaucoma de ángulo cerra do. Los pacientes que sigan tratamiento a largo plazo, especialmente aquellos tratados a dosis elevadas, deben ser monitorizados con especial atención, evaluán dose clínicamente de forma periódica para determinar si es posible una disminu ción de la dosis. En tratamientos con neurolépticos es posible el desarrollo de un sindrome neuroléptico maligno thipertermia, rigidez, fluctuaciones del estado de vigilia, inestabilidad del sistema nervioso autónomol. Los pacientes con un sindrome cerebral orgánico, retardo mental y aquellos con historia de abuso de opiáceos o alcohol presentan una incidencia mayor de casos con desenlace mortal. El tratamiento del síndrome neuroléptico maligno se basa en la retirada del neuroléptico, tratamiento sintomático y medidas de soporte vital. Puede administrarse bromocriptina. Los síntomas pueden persistir durante un periodo superior a una semana desde la interrupción del tratamiento oral, y por un periodo más prolongado cuando el síndrome neuroléptico maligno se asocia a las formos depot del fármaco. INTERACCIONES: El decanoato de zuclopentixol puede potenciar el efecto sedante del alcohol y el efecto de los barbitúricos y otros depreso res del sistema nervioso central. los neurolépticos pueden incrementar o disminuir el efecto de algunos fármacos antihipertensivos. El efecto anthipertensivo de la guanetidina y otros fármacos con un mecanismo de acción semejante se ve disminuido por el decanoato de zuclopentixol. El uso concomitante de neurolépti$\cos$ y litio cumenta el riesgo de neurotoxicidad. Los antidepresivos triciclicos y los neurolépticos inhiben mutuamente su metabolismo. El decanoato de zuclopentixol puede disminuir el efecto de la levodopa y de fármacos adrenérgicos. Administrado junto a metoclopramida y piperacina aumenta el riesgo de reacciones extrapiramidales. EMBARAZO Y LACTANCIA: El decanooto de zuclopentixol no debe administrarse durante el embarazo, en aquellos en que beneficio esperado para la paciente supere el riesgo teórico para el feto. Los estudios de reproducción animal no han aportado evidencia de un aumento de la incidencia de daño fetal ni otros efectos deletéreos en el proceso de reproducción. Se desaconseja la lactancia materna en pacientes en tratamiento con zuclopentixol. EFECTOS SOBRE LA CAPACIDAD PARA CONDUCIR O UTILIZAR MAQUINARIA: El decanoato de zuclopentixol es un fármaco sedante. Los pacientes que reciben fármacos psicotropos pueden presentar alteraciones en la capacidad de concentración y estado de atención, sea debido a la enfermedad de base, a la medicación o a ambas. Los pacientes que reciban tratamiento con zuclopentixol deberán tenerlo en cuenta en caso de que deban conducir automóviles o manejar maquinaria peligrosa. REACCIONES ADVERSAS. La mayoría de las reacciones adversas son dependientes de la dosis. La frecuencia y severidad de las mismas son más pronunciadas en la fase inicial del tratamiento y disminuyen durante el tratamiento crónico. En ensayos clínicos se han descrito las siguientes reacciones adversas. Dosis de $200-400 \mathrm{mg} / 2-4$ semanas. Frecuentes ( $\geq 5 \%$ ). Sistema nervioso central y periférico: Pueden presentarse efectos extrapiramidales, especialmente durante los primeros días después de una inyección y en la fase inicial de tratamiento. En la mayoría de los casos las reacciones adversas pueden controlarse satisfactoriamente con una reducción de la dosis y/o administrando fármacos antiparkinsonianos. Se desaconseja el uso profiláctico rutinario de fármacos antiparkinsonianos. En casos de acatisia persistente puede ser de utilidad la administración de una benzodiacepina o propranolol. Se ha descrito la aparición de los siguientes síntomas extrapiramidales: parkinsonismo (1 $1 \%)$, hipercinesia $(=$ acatisia) $(3,8 \%)$, distonía $(1,2 \%)$, hipocinesia $(2,4 \%)$, temblor $(0,8 \%)$, rigidez $10,6 \%)$. Psiquiatricos: Somnolencia (10\%): Aparato digestivo: Sequedad de boca $(6 \%)$. Generales: Fatiga(6\%). Infrecuentes (1-4\%). Sistema nervioso central y periférico: Mareo $(2 \%)$, cefalea $(1,7 \%)$. Alteraciones oculares: Alteraciones en la acomodación $(1,4 \%)$. Aparato digestivo: Aumento de la salivación $(1,4 \%)$, constipación $(1,2 \%)$ dispepsia/náuseas/vómitos $(1,7 \%)$. Sistema cardiovascular, general: Hipotensión postural $(1 \%)$. Raras $(<1 \%)$. Piel y anexos: Sudoración aumentada, rash. Sistema metabólico-nutricional: Aumento de peso. Sistema cardiovascular: Taquicardia/palpitaciones. Aparato urogenital- Incontinencia/retención urinarias. Dosis elevados (mínimo de $360 \mathrm{mg}$ por sernana, hasta $1600 \mathrm{mg}$ por inyección). Frecuentes (>5\%). Sistema nevvioso central y periférico: Trastornos extrapiramidales: hipocinesia $(22 \%)$, temblor( $19 \%$, parkinsonismo $(8 \%)$, hipercinesia $(3,8 \%)$, distonía $(1,5 \%)$. Mareo $(9 \%)$. Alteraciones oculares: Alteraciones en la acomodación (6\%). Psiquiatricos: Aumento del apetito (18\%), somnolencia (14\%). Aparato digestivo: Constipación (12\%), aumento de la salivación $(8 \%)$, sequedad de boca (8\%). Sistema cardiovascular, general: Hipotensión (10\%). Generales: Astenia (19\%). Infrecuentes (1-4\%). Sistema nervioso central y periférico: Convulsiones $(1,5 \%)$, cefalea $(1,5 \%)$. Psiquiatricos: Alteraciones de la libido $(1,5 \%)$. Aparato digestivo: dispepsia/náuseas/vómitos (3\%). Trastornos de la reproduc ción, Femeninos: Amenorrea/galactorrea (3\%) (1000-1600 mg por inyección). En algunos pacientes en tratamientos a largo plazo puede aparecer discinesia tardia. Los fármacos antiparkinsonianos no alivian estos síntomas, y en algunos casos pueden intensificarlos. Se recomienda en estos casos una reducción de la dosis 0 , en caso de ser posible, la interrupción del tratamiento. Se ha comunicado la aparición de sindrome neuroléptico maligno. lgualmente se ha reportado con poca frecuencia alteraciones en las pruebas de función hepática, en todos los casos leves y transitorias. Se han notificado casos aislados de hepatitis y/o ictericia en los que el zuclopentixol podría haber estado involucrado. SOBREDOSIFICACIÓN: Debido a la forma de administración del fármaco es dificil que se produzca sobredosificación del mismo. Sintomas: Somnolencia, coma, síntomas extrapiramidales, convulsiones, shock hiper o hipotermia. Tratamiento: El trata miento es sintomático y de soporte. Se establecerán medidas de soporte de los sistemas cardiovascular y respiratorio. No debe utilizarse epinefrina, debido al riesgo de reducción de la presión aterial. En caso de presentarse convulsiones, deberán ser tratados con diazepam. Los síntomas extrapiramidales pueden controlarse con biperideno. INCOMPATIBILIDADES: El decanoato de zuclopentixol solo se mezclará con el acetato de zuclopentixol, ya que ambos estan disueltos en aceite vegetal. El decanoato de zuclopentixol no deberá mezclarse con formulaciones depol que presentan como vehículo aceite de sésamo, pues la combinación daría lugar a cambios importantes en las propiedades farmacocinéticas de los preparados. PRECAUCIONES DE CONSERVACIÓN: Debe almacenarse a temperatura inferior a 15\%. INSTRUCCIONES DE USO/ MANIPULACIÓN: Ninguna. NOMBRE O RAZÓN SOCIAL Y DOMICILIO PERMANENTE O SEDE SOCIAL DEL TITULAR DE LA AUTORIZACION: Lundbeck España, S.A. Av. Diagonal, 605, $4^{\circ} 6^{\circ} .08028$ Barcelona. CON RECETA MEDICA. PRESENTACION Y PVP IVA (4\%): Clopixol ${ }^{8} 10 \mathrm{mg}$. Envase conteniendo 30 comprimidos, 1.248 pts. Envase conteniendo 50 comprimidos, $2.084 \mathrm{ps}^{\dagger}$. Clopixol ${ }^{8} 25 \mathrm{mg}$. Envase conteniendo 30 comprimidos 2.718 pts. Clopixol ${ }^{B}$ Gotas. Envase conteniendo $20 \mathrm{ml}, 1.905$ pts. Clopixol ${ }^{\circledR}$ Acufase. Envase conteniendo una ampolla de 1 $\mathrm{ml} 1.981$ pts. Envase conteniendo 10 ampollas de $1 \mathrm{ml}, 18.440$ pts. Clopixol Depot. Envase conteniendo una ampolla de $1 \mathrm{ml}, 1.669$ pts. CONDICIONES DE PRESTACIÓN FARMACÉUTICA DEL S.N.S. Á esta especialidad le corresponde APORTACIÓN REDUCIDA TEXTO REVISADO EN NOVIEMBRE 1995. 

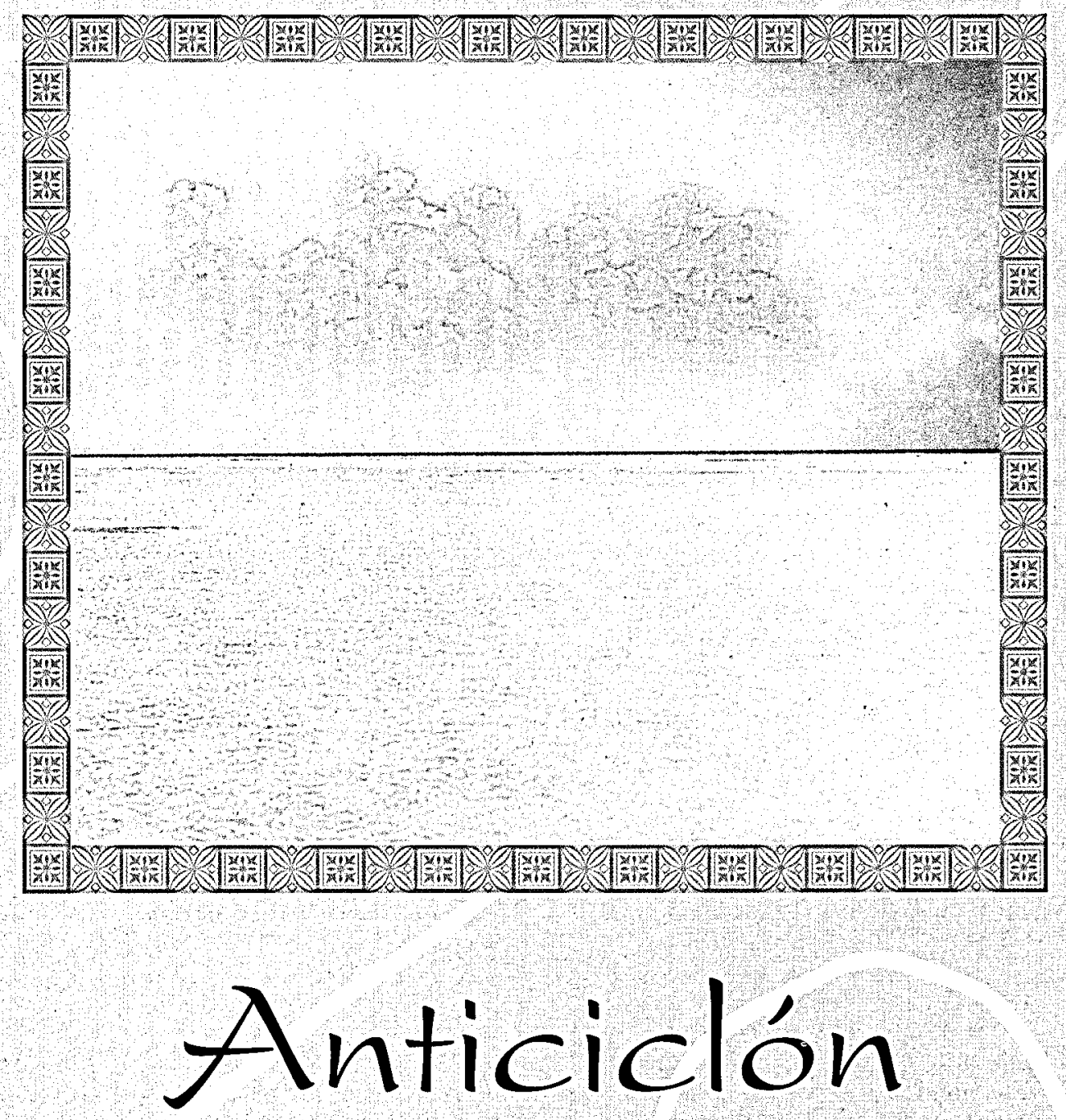

\section{SITUACIÓN BAJO CONTROL DEL PACIENTE ESQUIZOFRÉNICO}

El paciente esquizofrénico, al iqual que la naturaleza, puede perder el control en cualquier momenio. Clopixol permite controlar la esquizofrenia en la tase de manienimiento. Porque gracias a la presentación en inyectables depot se mejora el cumplimiento de la medicación por parie del paciente previniendo las recidivas. Además, al conirolar la enfermedad, se mejora la calidad de vida del paciente y de su entorno familiar. Clopixol depot, todo un aniciclón para la esquizolrenia.

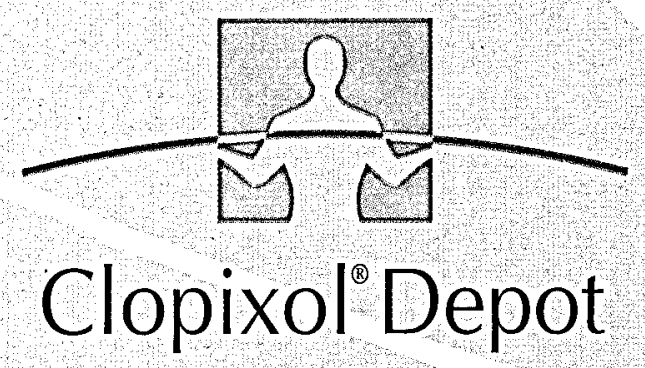

zuclopentixol

EL ANTICICLÓN DEPOT

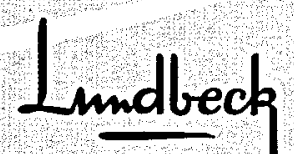

Research for a better life 